\title{
Micromechanics modeling for the stiffness and strength properties of glass fibers/CNTs/epoxy composites
}

\author{
M. Kim, F. A. Mirza \& J. I. Song \\ School of Mechatronics Engineering, \\ Changwon National University, South Korea
}

\begin{abstract}
This research explores micromechanics modeling for the prediction of stiffness and strength properties of nanocomposites consisting of randomly oriented carbon nanotubes (CNTs) and an epoxy matrix, and multiscale composites consisting of unidirectional glass fibers, randomly orientated CNTs, and an epoxy matrix. For stiffness properties, including Young's modulus, Poisson's ratio, and shear modulus, of CNTs/epoxy composites and glass fiber/CNTs/epoxy composites, the Mori-Tanaka model and Halpin-Tsai equations were used. To obtain the strength properties of the composites, including tensile and compressive strength, several empirical equations were employed. The estimated mechanical properties of nanocomposites were used as matrix properties in the micromechanical models for multiscale composites. The results showed that the stiffness and strength properties of nanocomposites and multiscale composites were improved by integrating CNTs in the systems.
\end{abstract}

Keywords: carbon nanotubes, fiber reinforced composites, micromechanics modeling, mechanical properties.

\section{Introduction}

The extraordinary physical properties of carbon nanotubes (CNTs) have encouraged many researchers to study CNT/polymer nanocomposites. de Viollia et al. [1] showed improvement of the tensile modulus and strength with the inclusion of 0.1 wt.\% of CNTs in epoxy resin. The improvement of Young's modulus, yield strength, strain to failure, and fracture toughness were reported 
with the integration of CNTs in a polymer matrix [2-4]. Adding 5 wt.\% of MWNTs in an epoxy enhanced the shear strength by $45.6 \%$ in Hsiao et al. [5]. Nanoparticles can also be mixed in fiber-reinforced resin to improve the matrixdominated properties, such as the compressive and flexural strength of a composite [6, 7]. Gojny et al. [8] and Wichmann et al. [9] showed the improvement of interlaminar shear strength of glass-fiber-reinforced composites by nanoparticle modification in a polymer matrix.

In conjunction with experimental studies, prediction of the mechanical behavior of CNT-polymer composites using numerical models is necessary for their use in industrial structures. The Mori-Tanaka model [10] and Halpin-Tsai equations [11] are popular methods for estimating the mechanical properties of fiber reinforced composites and are used widely to predict the mechanical properties of nanocomposites [12-16]. Using the Eshelby-Mori-Tanaka method, Ashrafi et al. [13] showed that CNTs are able to enhance the axial Young's modulus and longitudinal wave velocity of nanocomposite beams. Odegard et al. [14] and Ashrafie and Hubert [15] linked the atomistic simulations of nanostructured materials to continuum models of the corresponding bulk material to determine the properties of SWNT/polymer composites.

The Halpin-Tsai equations, based on the generalized self-consistent micromechanics solutions, are simple approximate forms for composites modeling developed by Hill $[17,18]$. There have been several research efforts to apply the Halpin-Tsai equations to CNT-reinforced polymer composites. To obtain Young's modulus and tensile strength of the MWNTs/phenolic composites, Yeh et al. [19] modified the Halpin-Tsai equation by employing an orientation factor and an exponential shape factor in the equation. Several modified Halpin-Tsai equations were used to obtain the tensile modulus of nanocomposites, including randomly oriented CNTs [2, 20, 21].

Several research works have been done to predict the mechanical properties of nanocomposites. However, there are few research works for obtaining the mechanical properties of multiscale composites using numerical models. In this study, the stiffness and strength properties of nanocomposites and multiscale composites were predicted using the Mori-Tanaka model, Halpin-Tsia equations, and several empirical equations. To obtain the stiffness and strength properties of multiscale composites, a methodology is presented by using the estimated stiffness and strength properties of nanocomposites as mechanical properties of the matrix in the micromechanics models for multiscale composites.

\section{Modeling}

Fig. 1 illustrates multiscale composites simulated in this research. The composites consist of unidirectional continuous glass fibers, random orientation CNTs, and an epoxy matrix.

Fig. 2 shows a schematic of modeling for nanocomposites and multiscale composites. For the stiffness properties of CNT-modified epoxy composites, the Mori-Tanaka model was used. To obtain the stiffness properties of multiscale composites, the Mori-Tanaka model and Halpin-Tsai equations were employed 


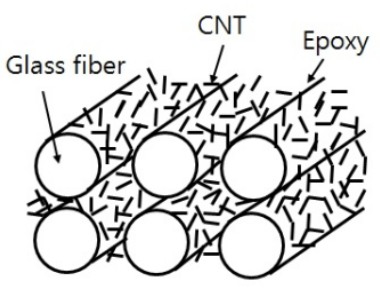

Figure 1: $\quad$ Multiscale composites.
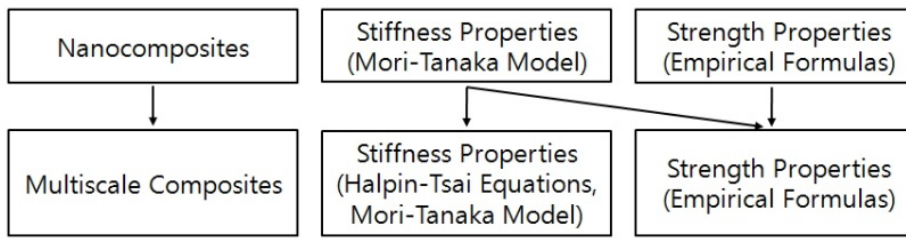

Figure 2: $\quad$ Schematic of modeling.

and the results of both models were compared. The two micromechanics models need the material properties of two constituent materials, reinforcement and the matrix. For the glass fibers/CNTs/epoxy matrix composites, the material properties of CNTs/epoxy composites from the Mori-Tanaka model were used as the matrix properties in the calculation of the two models, which makes the system multiscale consisting of nanoscale CNTs and microscale fibers. The stiffness properties of nanocomposites were also used for the prediction of the strength properties of multiscale composites as shown in fig. 2. In the relation of the strength properties of nanocomposites and multiscale composites, the estimated strength properties of nanocomposites were used as the strength properties of the matrix in multiscale composites.

In the simulation, several assumptions were made to simplify the problem. It was assumed that the interfacial bondings of the fiber-matrix and the CNTmatrix were perfect. Each CNT was assumed to be a straight rod with the same diameter and length and the same mechanical properties. Additionally, the dispersion of CNTs was perfect and there was no void in the matrix.

\subsection{Modeling for stiffness properties}

\subsubsection{Mori-Tanaka model and stiffness tensor}

The first step of this work is to obtain stiffness properties of CNT/epoxy nanocomposites. The stiffness properties of composites including, Young's moduli, Poisson's ratios, and shear moduli, can be obtained from the stiffness tensor [22]. The stiffness tensor $C_{i j k l}$ of a composite system, which contains noncolliding and homogeneously dispersed rigid rods with uniform aspect ratio, can be expressed as $[23,24]$ 


$$
\begin{aligned}
\mathbf{C}_{i j k l} & =B_{1}\left(a_{i j k l}\right)+B_{2}\left(a_{i j} \delta_{k l}+a_{k l} \delta_{i j}\right)+B_{3}\left(a_{i k} \delta_{j l}+a_{i l} \delta_{j k}+a_{j l} \delta_{i k}+a_{j k} \delta_{i l}\right) \\
& +B_{4}\left(\delta_{i j} \delta_{k l}\right)+B_{5}\left(\delta_{i k} \delta_{j l}+\delta_{i l} \delta_{j k}\right)
\end{aligned}
$$

where $\delta_{i j}$ is the Kronecker Delta ( $\delta_{i j}=1$ when $i=j$ and $\delta_{i j}=0$ when $i \neq j$ ); $a_{i j}$ and $a_{i j k l}$ are the second and fourth rank CNT orientation tensors (also called the moments of the distribution), respectively [23]; the five scalar constants, $\mathrm{B}_{i}$ 's, are obtained from the coefficients of the unidirectional stiffness tensor $[23,24]$. In this work, the unidirectional stiffness tensor was obtained using the MoriTanaka model [12-15, 25, 28], expressed as

$$
\overline{\mathbf{C}}_{i j k l}=\mathbf{C}^{m}+V_{f}\left\langle\left(\mathbf{C}^{f}-\mathbf{C}^{m}\right) \mathbf{A}^{f}\right\rangle\left(V_{m} \mathbf{I}+V_{f}\left\langle\mathbf{A}^{f}\right\rangle\right)^{-1}
$$

where $\mathbf{C}^{m}$ is the stiffness tensor of the matrix material; $\mathbf{C}^{f}$ is the stiffness tensor of the filler; $V_{m}$ and $V_{f}$ are the matrix and filler volume fractions, respectively; $\mathbf{I}$ is the identity tensor; $\mathbf{A}^{f}$ is the dilute mechanical strain concentration tensor for the filler given as the ratio between the average filler strain (or stress) and the corresponding average in the composite $[11,14]$

$$
\mathbf{A}^{f}=\left[\mathbf{I}+\mathbf{S}\left(\mathbf{C}^{m}\right)^{-1}\left(\mathbf{C}^{f}-\mathbf{C}^{m}\right)\right]^{-1}
$$

The tensor S, given by Eshelby [11], is called Eshelby's tensor whose elements are functions of the aspect ratio of an ellipsoidal inclusion and the Poisson's ratio of the matrix [13]. The terms enclosed with angle brackets in eqn (2) represent the average (or expectation) value of the term over all orientations.

\subsubsection{Halpin-Tsai equations}

To obtain stiffness properties of multiscale composites, Halpin-Tsai equations were used, which are based on the generalized self-consistent micromechanics solutions [17, 18]. Halpin and Kardos [18] reviewed the developments of HalpinTsai equations. The equations have been used for a variety of reinforcement geometries, including fibers, flakes, and ribbons. The general form of the HalpinTsai equations for aligned reinforcements is expressed as [17]

$$
\frac{P}{P_{m}}=\frac{1+\zeta \eta V_{r}}{1-\eta V_{r}}
$$

with

$$
\eta=\frac{\left(P_{r} / P_{m}\right)-1}{\left(P_{r} / p_{m}\right)+\zeta}
$$

where $P$ is composite property, such as $E_{11}, E_{22}, G_{12}, G_{23}$, and $v_{23} ; P_{r}$ is reinforcement property, such as $E_{r}, G_{r}$, and $v_{r} ; P_{m}$ is matrix property, such as $E_{m}$, $\mathrm{G}_{\mathrm{m}}$, and $v_{\mathrm{m}} ; \zeta$ is a measure of reinforcement geometry, packing geometry, and loading conditions; $V_{r}$ is reinforcement volume fraction (see e.g. [29] for complete Halpin-Tsai equations). 


\subsection{Modeling for strength properties}

Since predicting strength properties of composites is not as successful as predicting stiffness properties of the composites, many research efforts are active in this field. Several empirical equations for strength properties of composites are presented in Barbero [26]. The equations used in this research for the prediction of strength properties of nano and multiscale composites are listed in Appendix, eqns (A1)-(A7).

\section{Results and discussion}

Stiffness properties and ultimate strength properties of nanocomposites and multiscale composites were predicted in this work. As mentioned in Section 2, the nanocomposite properties were used in the calculation of Mori-Tanaka model and Halpin-Tsai equations for multiscale composites. The three constituent materials, glass fibers, epoxy matrix, and CNTs, were assumed to be isotropic materials. Young's moduli of glass fiber and epoxy were $72 \mathrm{GPa}$ and $3.0 \mathrm{GPa}$, respectively $[17,26]$. Poisson's ratios of glass fiber and epoxy were 0.2 and 0.3 , respectively. $450 \mathrm{GPa}$ and 0.3 were used for Young's modulus and Poisson's ratio of CNTs, respectively [27]. Each CNT have same geometry, $20 \mathrm{~nm}$ of diameter and $10 \mu \mathrm{m}$ of length. The fiber volume fraction of multiscale composites was 0.6 and the simulation was performed from 0 to $10 \mathrm{vol} \%$ of CNTs.

\subsection{Mechanical properties of nanocomposites}

\subsubsection{Stiffness properties of nanocomposites}

Fig. 3 shows the stiffness properties of nanocomposites including random orientation CNTs obtained by using eqn (1). Since tensile and shear moduli of CNTs are higher than epoxy, tensile and shear moduli of nanocomposites increase as CNT loading increases in the epoxy matrix. Poisson's ratio in fig. 3 (c) decreases with the increase of CNT loading. The inclusion of high modulus CNTs in random orientation may improve the stiffness of nanocomposites, resulting in the reduction of Poisson's ratio of nanocomposites.

\subsubsection{Strength properties of nanocomposites}

Fig. 4 shows tensile strength of nanocomposites obtained using eqn (A7). The strength increases as CNT loading increases. It seems that random orientation CNTs contribute to the improvement of tensile strength of nanocomposites. Since there seem no formulas available for compressive and inplane shear strength of randomly oriented composites [26], compressive and inplane shear strengths were assumed to be equal to and one-half of the tensile strength, respectively. 


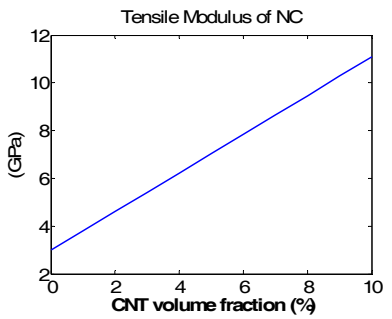

(a) Tensile modulus

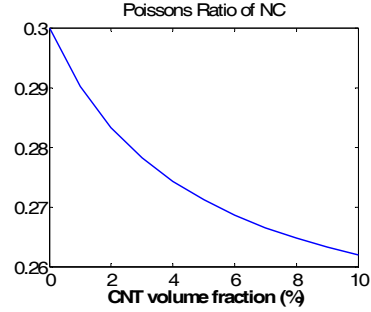

(c) Poisson's Ratio

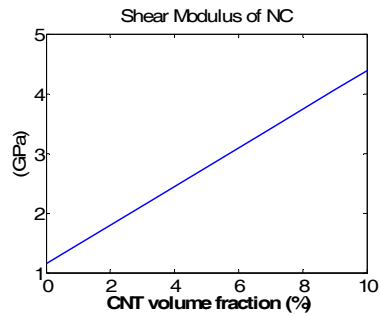

(b) Shear modulus

Figure 3: Stiffness properties of nanocomposites.

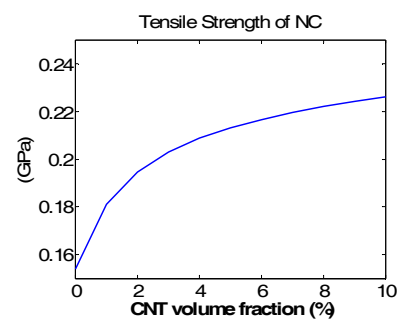

Figure 4: Tensile strength of nanocomposites.

\subsection{Mechanical properties of multiscale composites}

\subsubsection{Stiffness properties of multiscale composites}

Fig. 5 shows the stiffness properties of multiscale composites obtained by Halpin -Tsai equations (eqn (4)) and Mori-Tanaka model (eqn (2)). Young's and shear moduli increase as CNT loading increase, which is similar trend to the properties of nanocomposites. The modulus improvement ratios (between the moduli of 10 vol\%-CNT and 0 vol\%-CNT composites) of multiscale composites in figs 5 (a) and (b), however, are smaller than that of nanocomposites in fig 3 (a) because of the existence of fibers. Another interesting finding from fig. 5 is the improvements of tensile modulus in longitudinal and transverse directions. In fig. 5 (a), longitudinal tensile modulus of multiscale composites at $10 \mathrm{vol} \%$ of CNTs is $47.9 \mathrm{GPa}$ which is $7.4 \%$ improvement from $44.6 \mathrm{GPa}$ of tensile modulus at 0 vol. $\%$ of CNTs. Transverse tensile modulus $26.9 \mathrm{GPa}$ at 10 vol. $\%$ of CNTs is improved from the modulus $9.6 \mathrm{GPa}$ at 0 vol.\% of CNTs by 


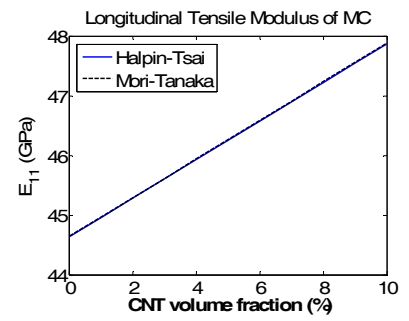

(a) Longitudinal tensile modulus

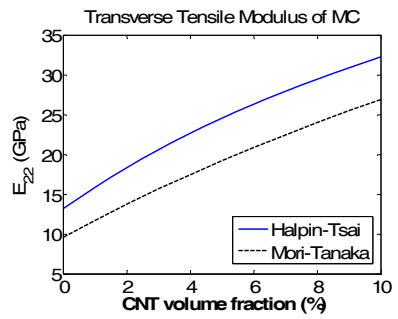

(b) Transverse tensile modulus

Figure 5: Tensile modulus of multiscale composites.

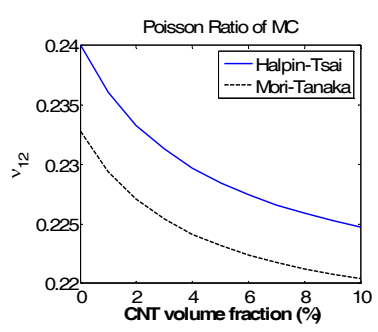

(a) Poisson's ratio $v_{12}$

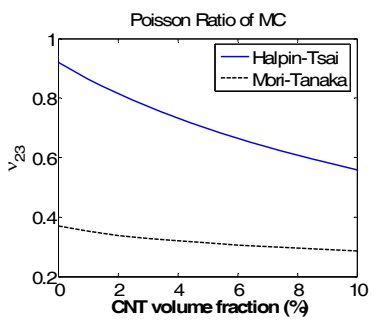

(a) Poisson's ratio $v_{23}$

Figure 6: Poisson's ratio of multiscale composites.

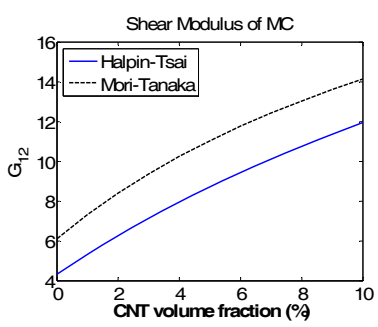

(a) Shear modulus $\mathrm{G}_{12}$

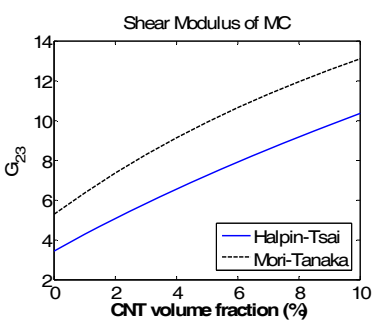

(b) Shear modulus $\mathrm{G}_{23}$

Figure 7: Shear modulus of multiscale composites.

$180.2 \%$. Since the matrix properties affect the mechanical properties of multiscale composites more in transverse direction than in longitudinal direction, the enhancement of transverse tensile modulus is more pronounced than longitudinal tensile modulus by the inclusion of CNTs. In fig. 5 (b), Halpin-Tsai equations generated higher tensile modulus in transverse direction than MoriTanaka model.

Fig. 6 shows Poisson's ratios of multiscale composites. Poisson's ratios of multiscale composites decrease with the increase of CNT loading. Since, in fig. 6 (b), the Poisson's ratio $v_{23}$ from Halpin-Tsai equations is higher than 0.5 , the result of Mori-Tanaka model seems more reasonable than that of Halpin-Tsai equations. Fig. 7 shows that shear modulus of multiscale composites. Shear modulus increases as CNT loading increases, which is similar to Young's 
modulus of multiscale composites. However, Mori-Tanak model generated higher shear modulus than Halpin-Tsai equations unlike the result of transverse Young's modulus of multiscale composites in fig. 5 (b).

\subsubsection{Strength properties of multiscale composites}

The strength properties of multiscale composites obtained using eqns (A1)-(A6) are shown in fig. 8. Longitudinal tensile strength increases linearly whereas transverse tensile strength increases nonlinearly in figs 8 (a) and (b). A similar trend is also shown in compressive strengths in both longitudinal and transverse directions (figs 8 (c) and (d)). The improvement of transverse tensile strength in fig. 8 (b) is more profound than that of longitudinal tensile strength in fig. 8 (a) like the tensile moduli in fig. 5 .

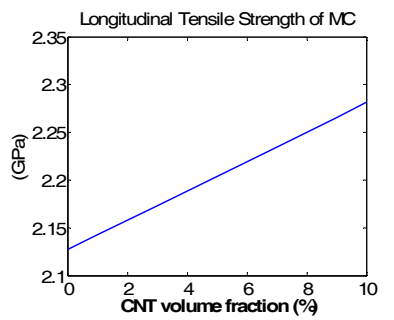

(a) Longitudinal tensile strength

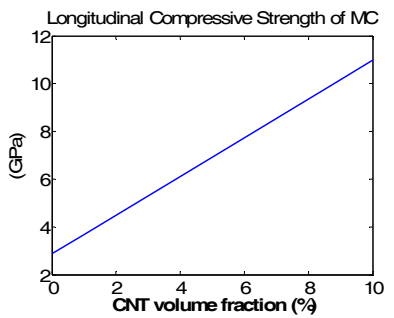

(c) Longitudinal compressive strength

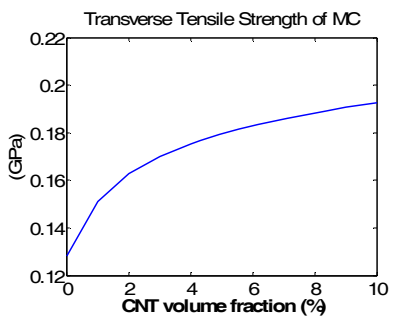

(b) Transverse tensile strength

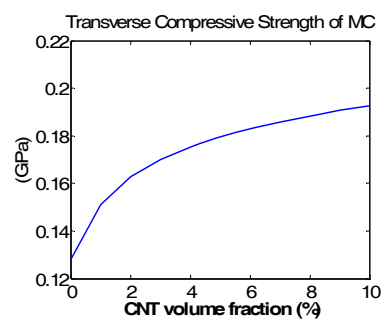

(d) Transverse compressive strength

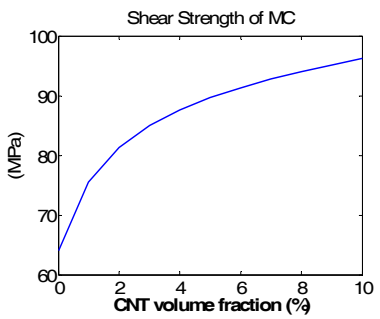

(e) Inplane shear strength

Figure 8: $\quad$ Strength properties of multiscale composites. 


\section{Conclusion}

Stiffness properties and ultimate strength properties of nanocomposites and multiscale composites were predicted using Mori-Tanaka model, Halpin-Tsai equations, and several empirical equations. The stiffness properties of nanocomposites were obtained using Mori-Tanaka micromechanics and the obtained properties were used for the prediction of stiffness and strength properties of multiscale composites. The strength properties of nanocomposites were also used as strength properties of matrix in the calculations for strength properties of multiscale composites.

The result showed that Young's modulus and shear modulus of nanocomposites increase as the CNT loading increases while the integration of CNTs reduced Poisson's ratio of nanocomposites. CNT modification enhanced the tensile strength of nanocomposites as well.

Stiffness and strength properties of multiscale composites have similar trend to the properties of nanocomposites, showing improved properties by CNT integration. Based on Poisson's ratio $v_{23}$ of multiscale composites, Mori-Tanaka method seems better mechanical property prediction method than Halpin-Tsai equations.

\section{Appendix}

In this work, the empirical formulas in Barbero [26] were employed for ultimate strength prediction. For longitudinal tensile strength, the composites were assumed to break when the stress in the filler reaches filler strength. With this assumption, longitudinal tensile strength can be expressed as [26]

$$
F_{1 t}=\sigma_{\mathrm{fa}}\left[V_{\mathrm{f}} \frac{E_{\mathrm{m}}}{E_{\mathrm{f}}}\left(1-\mathrm{V}_{\mathrm{f}}\right)\right]
$$

where $\sigma_{f a}$ is tensile strength of filler; $V_{f}$ is filler volume fraction; $E_{m}$ and $E_{f}$ are Young's moduli of epoxy matrix and filler, respectively.

The empirical formula used for transverse tensile strength is [26]

$$
\mathrm{F}_{2 \mathrm{t}}=\sigma_{\mathrm{mu}} \mathrm{C}_{\mathrm{v}}\left[1+\left(\mathrm{V}_{\mathrm{f}}-\sqrt{\mathrm{V}_{\mathrm{f}}}\right)\left(1-\frac{\mathrm{E}_{\mathrm{m}}}{\mathrm{E}_{\mathrm{f}}}\right)\right]
$$

where $\sigma_{\mathrm{mu}}$ is tensile strength of matrix, $\mathrm{C}_{\mathrm{v}}$ is empirical reduction coefficient on account of voids which is expressed as

$$
C_{v}=1-\sqrt{\frac{4 V_{v}}{\pi\left(1-V_{f}\right)}}
$$

where $\mathrm{V}_{\mathrm{v}}$ is void volume fraction. If there is no void in the system, $\mathrm{C}_{\mathrm{v}}$ will be 1.0 .

Compressive strength of unidirectional filler composites in longitudinal direction is lower than the tensile strength of the composites [26]. For 
compressive strength of the composites, buckling stress was used in this study, which is one critical failure mode in longitudinal compression. The buckling stress longitudinal compression is expressed as [26]

$$
\mathrm{F}_{1 \mathrm{c}}=\frac{\mathrm{G}_{\mathrm{m}}}{1-\mathrm{V}_{\mathrm{f}}}
$$

Transverse compressive strength may be obtained using eqn (A2), replacing the bulk tensile strength of the matrix $\sigma_{\mathrm{mu}}$ by the bulk compressive strength of the matrix $\sigma_{\text {muc }}[26]$. Transverse compressive strength is

$$
\mathrm{F}_{2 \mathrm{c}}=\sigma_{\mathrm{muc}} \mathrm{C}_{\mathrm{v}}\left[1+\left(\mathrm{V}_{\mathrm{f}}-\sqrt{\mathrm{V}_{\mathrm{f}}}\right)\left(1-\frac{\mathrm{E}_{\mathrm{m}}}{\mathrm{E}_{\mathrm{f}}}\right)\right]
$$

Since compressive strength of matrix is higher than tensile strength of matrix, $\sigma_{\text {muc }}$ was assumed to be twice of $\sigma_{\mathrm{mu}}$ in this work.

For inplane shear strength, an equation similar to eqn (A2) is used. Tensile strength $\sigma_{\mathrm{mu}}$ and Young's modulus E of matrix and filler in eqn (A2) were replaced by shear strength of matrix $\tau_{\mathrm{mu}}$ and shear moduli of matrix and filler. The inplane shear strength used in this research is [26]

$$
\mathrm{F}_{6}=\tau_{\mathrm{mu}} \mathrm{C}_{\mathrm{v}}\left[1+\left(\mathrm{V}_{\mathrm{f}}-\sqrt{\mathrm{V}_{\mathrm{f}}}\right)\left(1-\frac{\mathrm{G}_{\mathrm{m}}}{\mathrm{G}_{\mathrm{f}}}\right)\right]
$$

Since there seems no equation available for random orientation chopped strand reinforced composites, empirical equations for chopped strand mat were used for chopped strand composites. The tensile strength of random orientated and chopped strand mat $\mathrm{F}_{\mathrm{csm}-\mathrm{t}}$ may be expressed as [26]

$$
\begin{aligned}
& F_{c s m-t}=\frac{4 \alpha F_{2 t}}{\pi}\left[1+\frac{1}{2} \ln \left(\frac{F_{1 t}}{\alpha^{2} F_{2 t}}\right)\right] \text { for } \alpha \leq \sqrt{\frac{F_{1 t}}{F_{2 t}}} \\
& F_{c s m-t}=\frac{4 \alpha F_{2 t}}{\pi} \sqrt{\frac{F_{1 t}}{F_{2 t}}} \text { for } \alpha>\sqrt{\frac{F_{1 t}}{F_{2 t}}}
\end{aligned}
$$

where $\alpha=\frac{\mathrm{F}_{6}}{\mathrm{~F}_{2 \mathrm{t}}}$.

Compressive strength of random orientation chopped strand composites was assumed to be equal to the tensile strength $\mathrm{F}_{\mathrm{csm}-\mathrm{t}}$ and shear strength of chopped strand composites was assumed to be half of $\mathrm{F}_{\mathrm{csm}-\mathrm{t}}$.

\section{References}

[1] de Villoria, R.G., Miravete, A., Cuartero, J., Chiminelli, A., Tolosana, N., Mechanical properties of SWNT/epoxy composites using two different curing cycles, Composites: Part B, 37, pp. 273-277, 2006.

[2] Gojny, F.H., Wichmann, M.H.C, Köpke, U., Fiedler, B., Schulte, K., Carbon nanotube-reinforced epoxy-composites: enhanced stiffness and fracture toughness at low nanotube content, Composite Science and Technology, 64, pp. 2363-2371, 2004. 
[3] Thostenson, E.T., Chou, T.W., Processing-structure-multi-functional property relationship in carbon nanotube/epoxy composites, Carbon, 44, pp. 3022-3029, 2006.

[4] Allaoui, A., Bai, S., Cheng, H.M., Bai, J.B., Mechanical and electrical properties of a MWNT/epoxy composite, Composites Science and Technology, 62, pp. pp. 1993-1998, 2002.

[5] Hsiao, K.T., Alms, J., Advani, S.G., Use of epoxy/multiwalled carbon nanotubes as adhesives to join graphite fibre reinforced polymer composites, Nanotechnology, 14, pp. 791-793, 2003.

[6] Vlasveld, D.P.N., Bersee, H.E.N, Picken S J. Nanocomposite matrix for increased fibre composite strength, Polymer, 46, pp. 10269-10278, 2005.

[7] Iwahori, Y., Ishiwata, S., Sumizawa, T., Ishikawa, T., Mechanical properties improvements in two-phase and three-phase composites using carbon nano-fiber dispersed resin, Composites: Part A, 36, pp. 1430-1439, 2005.

[8] Gojny, F.H., Wichmann, M.H.G., Fiedler, B., Bauhofer, W., Schulte, K., Influence of nano-modification on the mechanical and electrical properties of conventional fibre-reinforced composites, Composites: Part A, 36, pp. $1525-1535,2005$.

[9] Wichmann, M.H.G., Sumfleth, J., Gojny, F.H., Quaresimin, M., Fiedler, B., Schulte, K., Glass-fibre-reinforced composites with enhanced mechanical and electrical properties - Benefits and limitations of a nanoparticle modified matrix, Engineering Fracture Mechanics, 73, pp. 2346-2359, 2006.

[10] Mori, T., Tanaka, K., Average stress in matrix and average elastic energy of materials with misfitting inclusions, Acta Metallurgica, 21, pp. 571-4, 1973.

[11] Eshelby, J.D., The determination of the elastic field of an ellipsoidal inclusion, and related problems. Proceedings of the Royal Society of London, Series A, A241, pp. 376-96, 1957.

[12] Tucker III, C.L., Liang, E., Stiffness prediction for unidirectional shortfibercomposites: Review and evaluation, Composites Science and Technology, 59, pp. 655-671, 1999.

[13] Ashrafi, B., Hubert, P., Vengallatore, S., Carbon nanotube-reinforced composites as structural materials for microactuators in microelectromechanical systems, Nanotechnology, 17, pp. 4895-4903, 2006.

[14] Odegard, G.M., Gatesb, T.S., Wisea, K.E., Parka, C., Siochic, E.J., Constitutive modeling of nanotube-reinforced polymer composites, Composites Science and Technology, 63, pp. 1671-1687, 2003.

[15] Ashrafi, B., Hubert, P., Modeling the elastic properties of carbon nanotube array/polymer composites, Composites Science and Technology, 66, pp. 387-396, 2006

[16] Kim, M., Park, Y.B., Okoli, O.I., Zhang, C., Processing characterization and modeling of carbon nanotube-reinforced multiscale composites. Composites Science and Technology, 69(3-4), pp. 335-342, 2009 
[17] Mallick, P.K., Fiber-reinforced composites, $2^{\text {nd }}$ edition, Marcel Dekker, Inc., 1993.

[18] Halpin, J.C., Kardos, J.L., The Halpin-Tsai equations: A review, Polymer Engineering and Science, 16(5), pp. 344-352, 1976.

[19] Yeh, M.K., Tai, N.H., Liu, J.H., Mechanical behavior of phenolic-based composites reinforced with multi-walled carbon nanotubes, Carbon, 44, pp. 1-9, 2006.

[20] Peeterbroeck, S., Breugelmans, L., Alexandre, M., BNagy, J., Viville, P., Lazzaroni, R., et al., The influence of the matrix polarity on the morphology and properties of ethylene vinyl acetate copolymers-carbon nanotube nanocomposites. Compos Sci Technol, 67, pp. 1659-1665, 2007.

[21] Thostenson, E.T., Chou, T.W., On the elastic properties of carbon nanotube-based composites: modeling and characterization. J Phys D: Appl Phys, 36, pp. 573-583, 2003.

[22] Jones, R.M., Mechanics of composite materials, $2^{\text {nd }}$, Taylor \& Francis, 1999.

[23] Advani, S.G., Tucker, C.L., The Use of Tensors to Describe and Predict Fiber Orientation in Short Fiber Composites, Journal of Rheology, 31(8), pp. 751-784, 1987.

[24] Jack, D.A., Smith, D.E., Elastic Properties of Short-Fiber Polymer Composites, Derivation and Demonstration of Analytical Forms for Expectation and Variance from Orientation Tensors, Journal of Composite Materials, 42(3), pp. 277-308, 2008.

[25] Kim, M., Modeling, Manufacturing and Characterization of Nanocomposites and Multiscale Composites, $\mathrm{PhD}$ Dissertation, Florida State University, Department of Industrial and Manufacturing Engineering, 2009.

[26] Barbero, E.J., Introduction to Composite Materials Design, New York NY, Taylor \& Francis, 1999.

[27] Shao, L.H., Luo, R.Y., Bai, S. L., Wang, J., Prediction of effective moduli of carbon nanotube-reinforced composites with waviness and debonding, Composite structures, 87(3), pp. 274-281, 2009.

[28] Beneveniste, Y., A new approach to the application of Mori-Tanaka's theory in composite materials, Mechanics of Materials, 6, pp. 147-157, 1987

[29] Caselman, E., Elastic property prediction of short fiber composites using a uniform mesh finite element method, Master Thesis, University of Missouri - Columbia, Mechanical \& Aerospace Engineering, 2007 